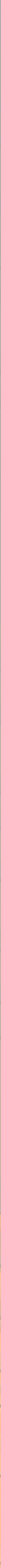




\title{
Interpolation for restricted tangent bundles of general curves
}

\author{
Eric Larson
}

Let $q_{1}, q_{2}, \ldots, q_{n} \in \mathbb{P}^{r}$ be a general collection of points, and $\left(C, p_{1}, p_{2}, \ldots, p_{n}\right)$ a general marked curve of genus $g$. We determine when there exists a nondegenerate degree- $d$ map $f: C \rightarrow \mathbb{P}^{r}$ such that $f\left(p_{i}\right)=q_{i}$ for all $i$. This is a consequence of our main theorem, which states that the restricted tangent bundle $f^{*} T_{\mathbb{p} r}$ of a general curve of genus $g$, equipped with a general degree- $d$ map $f$ to $\mathbb{P}^{r}$, satisfies the property of interpolation, i.e., that for a general effective divisor $D$ of any degree on $C$, either $H^{0}\left(f^{*} T_{\mathbb{p} r}(-D)\right)=0$ or $H^{1}\left(f^{*} T_{\mathbb{p} r}(-D)\right)=0$. We also prove an analogous theorem for the twist $f^{*} T_{\mathbb{p r}}(-1)$.

\section{Introduction}

The goal of this paper is to answer the following basic question about incidence conditions for curves.

Question 1.1. Fix a general marked curve $\left(C, p_{1}, p_{2}, \ldots, p_{n}\right)$ of genus $g$, and $n$ general points $q_{1}, q_{2}, \ldots, q_{n} \in \mathbb{P}^{r}$. When does there exist a nondegenerate degree- $d$ map $f: C \rightarrow \mathbb{P}^{r}$ so that $f\left(p_{i}\right)=q_{i}$ for all $i$ ?

(An analogous question when $\left(C, p_{1}, p_{2}, \ldots, p_{n}\right)$ is allowed to vary in moduli was recently answered in [Atanasov et al. 2016] for curves with nonspecial hyperplane section.)

In order for there to exist any nondegenerate degree- $d$ maps $f: C \rightarrow \mathbb{P}^{r}$, the Brill-Noether theorem [Griffiths and Harris 1980] states that the Brill-Noether number

$$
\rho(d, g, r):=(r+1) d-r g-r(r+1)
$$

must be nonnegative; so we assume this is the case for the remainder of the paper. In this case, writing $\operatorname{Map}_{d}\left(C, \mathbb{P}^{r}\right)$ for the space of nondegenerate degree- $d$ maps $C \rightarrow \mathbb{P}^{r}$, the Brill-Noether theorem additionally gives

$$
\operatorname{dim} \operatorname{Map}_{d}\left(C, \mathbb{P}^{r}\right)=\rho(d, g, r)+\operatorname{dim} \operatorname{Aut} \mathbb{P}^{r}=(r+1) d-r g+r .
$$

MSC2010: $14 \mathrm{H} 99$.

Keywords: restricted tangent bundle, interpolation. 
Thus, the answer to our main question can only be positive when

$$
(r+1) d-r g+r-r n \geq 0 .
$$

Our main theorem will imply (as an immediate consequence) that, conversely, the answer to our main question is positive when the above inequality holds. To state the main theorem, we first need to make a definition.

Definition 1.2. We say that a vector bundle $\mathcal{E}$ on a curve $C$ satisfies interpolation if it is nonspecial (i.e., $H^{1}(\mathcal{E})=0$ ), and for a general effective divisor $D$ of any degree $d \geq 0$, either

$$
H^{0}(\mathcal{E}(-D))=0 \quad \text { or } \quad H^{1}(\mathcal{E}(-D))=0 .
$$

For $C$ reducible, we require that the above holds for an effective divisor $D$ which is general in some (not every) component of $\operatorname{Sym}^{d} C$ (for each $d \geq 0$ ).

With this notation, we state our main result.

Theorem 1.3. Let $C$ be a general curve of genus $g$, equipped with a general nondegenerate map $f: C \rightarrow \mathbb{P}^{r}$ of degree $d$. Then $f^{*} T_{\mathbb{P} r}$ satisfies interpolation.

To see that this theorem implies a positive answer to the main question subject to the inequality (1), we first note that by basic deformation theory, the map $\operatorname{Map}_{d}\left(C, \mathbb{P}^{r}\right) \rightarrow\left(\mathbb{P}^{r}\right)^{n}$, defined via $f \mapsto\left(f\left(p_{i}\right)\right)_{i=1}^{n}$, is smooth at $f$ provided that $H^{1}\left(f^{*} T_{\mathbb{P} r}\left(-p_{1}-\cdots-p_{n}\right)\right)=0$. Note that the left-hand side of (1) equals $\chi\left(f^{*} T_{\mathbb{P} r}\right)$. Consequently, it suffices to show, for $C$ a general curve of genus $g$, equipped with a general nondegenerate map $f: C \rightarrow \mathbb{P}^{r}$, that $H^{1}\left(f^{*} T_{\mathbb{P}} r\left(-p_{1}-\cdots-p_{n}\right)\right)$ vanishes whenever $\chi\left(f^{*} T_{\mathbb{P} r}\left(-p_{1}-\cdots-p_{n}\right)\right) \geq 0$. But this is immediate provided that $f^{*} T_{\mathbb{P} r}$ satisfies interpolation. We have thus shown the following corollary of our main theorem.

Corollary 1.4. Fix a general marked curve $\left(C, p_{1}, p_{2}, \ldots, p_{n}\right)$ of genus $g$, and $n$ general points $q_{1}, q_{2}, \ldots, q_{n} \in \mathbb{P}^{r}$. There exists a nondegenerate degree-d map $f: C \rightarrow \mathbb{P}^{r}$ such that $f\left(p_{i}\right)=q_{i}$ for all $i$ if and only if $\rho(d, g, r) \geq 0$ and

$$
(r+1) d-r g+r-r n \geq 0 .
$$

Additionally, we prove a similar theorem for the twist of the tangent bundle; this answers a similar question, where $d$ of the $n$ points are constrained to lie on a hyperplane.

Theorem 1.5. Let $C$ be a general curve of genus $g$, equipped with a general nondegenerate map $f: C \rightarrow \mathbb{P}^{r}$ of degree $d$. Then $f^{*} T_{\mathbb{P r}}(-1)$ satisfies interpolation if and only if

$$
d-r g-1 \geq 0
$$


To see the "only if" direction, first note that since $C$ is nondegenerate, the restriction map $H^{0}\left(T_{\mathbb{P} r}(-1)\right) \rightarrow H^{0}\left(f^{*} T_{\mathbb{P} r}(-1)\right)$ is injective, so $\operatorname{dim} H^{0}\left(f^{*} T_{\mathbb{P} r}(-1)\right) \geq$ $\operatorname{dim} H^{0}\left(T_{\mathbb{P} r}(-1)\right)=r+1$. If $d-r g-1<0$, then $\chi\left(f^{*} T_{\mathbb{P} r}(-1)\right)=d-r g+r<r+1$; consequently $H^{1}\left(f^{*} T_{\mathbb{P} r}(-1)\right) \neq 0$, so $f^{*} T_{\mathbb{P} r}(-1)$ does not satisfy interpolation. For the remainder of the paper, we will therefore assume $d-r g-1 \geq 0$ in Theorem 1.5. As before, Theorem 1.5 gives the "if" direction of the next result.

Corollary 1.6. Fix a general marked curve $\left(C, p_{1}, p_{2}, \ldots, p_{n}\right)$ of genus $g$, and $n$ points $q_{1}, q_{2}, \ldots, q_{n} \in \mathbb{P}^{r}$, which are general subject to the constraint that $q_{1}, q_{2}, \ldots, q_{d}$ lie on a hyperplane $H($ for $d \leq n)$. There exists a nondegenerate degree-d map $f: C \rightarrow \mathbb{P}^{r}$ such that $f\left(p_{i}\right)=q_{i}$ for all $i$ if and only if $\rho(d, g, r) \geq 0$ and

$$
(r+1) d-r g+r-r n \geq 0 \text { and } d-r g-1 \geq 0 .
$$

For the "only if" direction, we first compute the dimension of the space of degree- $d$ maps $\operatorname{Map}_{d}\left(\left(C, p_{1} \cup p_{2} \cup \cdots \cup p_{d}\right),\left(\mathbb{P}^{r}, H\right)\right)$, from $C$ to $\mathbb{P}^{r}$, which send $p_{1} \cup p_{2} \cup \cdots \cup p_{d}$ to $H$. Choose coordinates $\left[x_{1}: x_{2}: \cdots: x_{r+1}\right]$ on $\mathbb{P}^{r}$ so $H$ is given by $x_{r+1}=0$. Then any such map is given by $r+1$ sections $\left[s_{1}: s_{2}: \cdots: s_{r}: 1\right]$ of $\mathcal{O}_{C}\left(p_{1}+p_{2}+\cdots+p_{d}\right)$, where we write $s_{r+1}=1 \in H^{0}\left(\mathcal{O}_{C}\right) \subset H^{0}\left(\mathcal{O}_{C}\left(p_{1}+\cdots+p_{d}\right)\right)$ for the constant section. By taking the sections $s_{1}, s_{2}, \ldots, s_{r}$, we have identified $\operatorname{Map}_{d}\left(\left(C, p_{1} \cup p_{2} \cup \cdots \cup p_{d}\right),\left(\mathbb{P}^{r}, H\right)\right)$ as an open subset of $H^{0}\left(\mathcal{O}_{C}\left(p_{1}+\cdots+p_{d}\right)\right)^{r}$, so its dimension is $r(d+1-g)$. Therefore, in order for $f \mapsto\left(f\left(p_{i}\right)\right)_{i=1}^{n}$ to dominate $H^{d} \times\left(\mathbb{P}^{r}\right)^{n-d}$, we must also have

$$
r(d+1-g) \geq(r-1) d+r(n-d) \quad \Longleftrightarrow \quad(r+1) d-r g+r-r n \geq 0 .
$$

In addition, $f \mapsto\left(f\left(p_{i}\right)\right)_{i=1}^{d}$ must dominate $H^{d}$. But the fibers of this map have a free action of the $(r+1)$-dimensional group $\operatorname{Stab}_{H}\left(\right.$ Aut $\left.\mathbb{P}^{r}\right)$; we must therefore also have

$$
r(d+1-g) \geq(r-1) d+(r+1) \quad \Longleftrightarrow \quad d-r g-1 \geq 0 .
$$

Remark 1.7. For $k \geq 2$, the bundles $f^{*} T_{\mathbb{P} r}(-k)$ almost never satisfy interpolation. Namely, in the setting of Theorem 1.3, the bundle $f^{*} T_{\mathbb{P} r}(-2)$ satisfies interpolation if and only if either $(g, r)=(0,1)$ or $(d, g, r)=(2,0,2)$; and $f^{*} T_{\mathbb{P} r}(-k)$ never satisfies interpolation for $k \geq 3$.

For $r=1$, this can be seen by observing that $f^{*} T_{\mathbb{P}^{1}}(-k) \simeq \mathcal{O}_{C}(2-k)$ is a line bundle, so it satisfies interpolation if and only if it is nonspecial (see Proposition 4.7 of [Atanasov et al. 2016]); as $k \geq 2$, this only happens if $k=2$ and $C$ is rational $(g=0)$.

In general, if $f^{*} T_{\mathbb{P r}}(-k)$ satisfies interpolation,

$$
\chi\left(f^{*} T_{\mathbb{P}}(-k)\right)=(r+1) d-r g+r-k r d \geq 0 .
$$


When $r \geq 2$ and $k \geq 2$, this is only satisfied for $(d, g, r, k)=(2,0,2,2)$; conversely, one may easily check that $f^{*} T_{\mathbb{P}^{2}}(-2)$ satisfies interpolation for $f: \mathbb{P}^{1} \rightarrow \mathbb{P}^{2}$ a general degree-2 map (for instance by combining Theorem 1.3 with Proposition 4.12 of [Atanasov et al. 2016]).

The remainder of the paper will be devoted to the proof of Theorem 1.3 and Theorem 1.5 using inductive degeneration. In Section 2, we begin by explaining how to use degeneration to approach the main theorems, and how to work with the condition of interpolation on reducible curves. Then in Section 3, we prove the main theorems for rational curves by inductively degenerating the curve $C$ to a union $D \cup L$, where $L$ is a 1 -secant line to $D$. Finally in Section 4, we prove the main theorems for arbitrary genus by degenerating the curve $C$ to a union $D \cup R$, where $R$ is a rational normal curve meeting $D$ at $1 \leq s \leq r+2$ points.

\section{Preliminaries}

Lemma 2.1. The locus of $f: C \rightarrow \mathbb{P}^{r}$ in $\bar{M}_{g}\left(\mathbb{P}^{r}, d\right)$ for which $f^{*} T_{\mathbb{P} r}$ satisfies interpolation is open, and likewise for $f^{*} T_{\mathbb{p} r}(-1)$. Moreover, every component of these loci dominates $\bar{M}_{g}$.

In particular, to prove Theorem 1.3 (respectively, Theorem 1.5) for curves of degree $d$ and genus $g$, it suffices to exhibit one, possibly singular, nondegenerate $f: C \rightarrow \mathbb{P}^{r}$ of degree $d$ and genus $g$, for which $f^{*} T_{\mathbb{P} r}$ (respectively, $f^{*} T_{\mathbb{P} r}(-1)$ ) satisfies interpolation.

Proof. Since the vanishing of cohomology groups is an open condition, it follows that interpolation is an open condition as well. (For a more careful proof, see Theorem 5.8 of [Atanasov 2014].)

If $f^{*} T_{\mathbb{p} r}$ (respectively, $\left.f^{*} T_{\mathbb{p} r}(-1)\right)$ satisfies interpolation, then in particular $H^{1}\left(f^{*} T_{\mathbb{P} r}\right)=0$ (respectively, $\left.H^{1}\left(f^{*} T_{\mathbb{P} r}(-1)\right)=0\right)$. Since $H^{1}\left(f^{*} T_{\mathbb{P} r}(-1)\right)=0$ implies $H^{1}\left(f^{*} T_{\mathbb{P} r}\right)=0$, we know either way that $H^{1}\left(f^{*} T_{\mathbb{P}} r\right)=0$. This completes the proof as the obstruction to smoothness of $\bar{M}_{g}\left(\mathbb{P}^{r}, d\right) \rightarrow \bar{M}_{g}$ lies in $H^{1}\left(f^{*} T_{\mathbb{P} r}\right)$.

In order to work with reducible curves, it will be helpful to introduce the following somewhat more general variant on interpolation.

Definition 2.2. Let $\mathcal{E}$ be a rank $n$ vector bundle over a curve $C$. We say that a subspace of sections $V \subseteq H^{0}(\mathcal{E})$ satisfies interpolation if $\mathcal{E}$ is nonspecial and, for every $d \geq 0$, there exists an effective Cartier divisor $D$ of degree $d$ such that

$$
\operatorname{dim}\left(V \cap H^{0}(\mathcal{E}(-D))\right)=\max \{0, \operatorname{dim} V-d n\} .
$$

We now state for the reader the elementary properties of interpolation that we shall need. 
Lemma 2.3 [Atanasov et al. 2016, Proposition 4.5]. A vector bundle $\mathcal{E}$ satisfies interpolation if and only if its full space of sections $V=H^{0}(\mathcal{E}) \subseteq H^{0}(\mathcal{E})$ satisfies interpolation.

Proof sketch. The result follows by examining the long exact sequence

$$
0 \rightarrow H^{0}(\mathcal{E}(-D)) \rightarrow H^{0}(\mathcal{E}) \rightarrow H^{0}\left(\left.\mathcal{E}\right|_{D}\right) \rightarrow H^{1}(\mathcal{E}(-D)) \rightarrow H^{1}(\mathcal{E})=0 .
$$

A complete proof is given at the location cited; see also Definition 4.1 in the same reference.

Lemma 2.4 [Atanasov et al. 2016, Proposition 8.1]. Let $\mathcal{E}$ be a vector bundle on a reducible curve $X \cup Y$, and $D$ be an effective divisor on $X$ disjoint from $X \cap Y$. Assume that

$$
H^{0}\left(\left.\mathcal{E}\right|_{X}(-D-X \cap Y)\right)=0 .
$$

Let

$$
\mathrm{ev}_{X}: H^{0}\left(\left.\mathcal{E}\right|_{X}\right) \rightarrow H^{0}\left(\left.\mathcal{E}\right|_{X \cap Y}\right), \quad \mathrm{ev}_{Y}: H^{0}\left(\left.\mathcal{E}\right|_{Y}\right) \rightarrow H^{0}\left(\left.\mathcal{E}\right|_{X \cap Y}\right)
$$

denote the natural evaluation morphisms. Then $\mathcal{E}$ satisfies interpolation provided

$$
V=\operatorname{ev}_{Y}^{-1}\left(\operatorname{ev}_{X}\left(H^{0}\left(\left.\mathcal{E}\right|_{X}(-D)\right)\right)\right) \subseteq H^{0}\left(\left.\mathcal{E}\right|_{Y}\right)
$$

satisfies interpolation and has dimension $\chi\left(\left.\mathcal{E}\right|_{Y}\right)+\chi\left(\left.\mathcal{E}\right|_{X}(-D-X \cap Y)\right)$.

Proof sketch. As $H^{0}\left(\left.\mathcal{E}\right|_{X}(-D-X \cap Y)\right)=0$, restriction to $Y$ gives an isomorphism $H^{0}(\mathcal{E}(-D)) \simeq V$. Also, the final dimension statement implies $H^{1}(\mathcal{E}(-D))=0$. Therefore $\mathcal{E}(-D)$, and hence $\mathcal{E}$, satisfies interpolation.

Lemma 2.5 [Atanasov 2014, Theorem 8.1 and Section 3]. Let $\mathcal{E}$ be a vector bundle on an irreducible curve $C$, and $p \in C_{\mathrm{sm}}$ be a general point. If $\mathcal{E}$ satisfies interpolation, and $\left.\Lambda \subseteq \mathcal{E}\right|_{p}$ is a general subspace of any dimension, then

$$
\left\{\sigma \in H^{0}(\mathcal{E}):\left.\sigma\right|_{p} \in \Lambda\right\} \subseteq H^{0}(\mathcal{E})
$$

satisfies interpolation and has dimension $\max \{0, \chi(\mathcal{E})-\operatorname{codim} \Lambda\}$.

Proof sketch. Let $n=\operatorname{rk} \mathcal{E}$, and $D$ be a general effective divisor of any degree $d \geq 0$. Since $\mathcal{E}$ satisfies interpolation,

$$
\begin{aligned}
\operatorname{dim} H^{0}(\mathcal{E}(-D)) & =\max \{0, \chi(\mathcal{E})-d n\}, \\
\operatorname{dim} H^{0}(\mathcal{E}(-D-p)) & =\max \{0, \chi(\mathcal{E})-d n-n\} .
\end{aligned}
$$

These inequalities imply the restriction map $\left.H^{0}(\mathcal{E}(-D)) \rightarrow \mathcal{E}\right|_{p}$ is either injective or surjective, which (since $\Lambda$ is general) in turn implies the composition $\left.\left.H^{0}(\mathcal{E}(-D)) \rightarrow \mathcal{E}\right|_{p} \rightarrow \mathcal{E}\right|_{p} / \Lambda$ is either injective or surjective. Together with the first of the above equalities, this implies

$$
\operatorname{dim}\left\{\sigma \in H^{0}(\mathcal{E}(-D)):\left.\sigma\right|_{p} \in \Lambda\right\}=\max \{0, \chi(\mathcal{E})-\operatorname{codim} \Lambda-d n\} .
$$




\section{Rational curves}

In this section, we prove Theorem 1.3 in the case $g=0$.

Proposition 3.1. Let $L \subseteq \mathbb{P}^{r}$ be a line. Then $\left.T_{\mathbb{P}^{r}}\right|_{L} \simeq \mathcal{O}_{L}(2) \oplus \mathcal{O}_{L}(1)^{r-1}$, where the $\mathcal{O}_{L}(2)$ summand comes from the inclusion $\left.T_{L} \hookrightarrow T_{\mathbb{P} r}\right|_{L}$; in particular, $\left.T_{\mathbb{P} r}\right|_{L}$ and $\left.T_{\mathbb{P} r}\right|_{L}(-1)$ satisfy interpolation.

Proof. The first assertion follows from the exact sequence

$$
\left.0 \rightarrow T_{L} \simeq \mathcal{O}_{L}(2) \rightarrow T_{\mathbb{P} r}\right|_{L} \rightarrow N_{L / \mathbb{P} r} \simeq \mathcal{O}_{L}(1)^{r-1} \rightarrow 0 .
$$

(We have $N_{L / \mathbb{P} r} \simeq \mathcal{O}_{L}(1)^{r-1}$ since $L$ is the complete intersection of $r-1$ hyperplanes.)

The second assertion follows from the first by inspection (or alternatively using Proposition 3.11 of [Atanasov 2014]).

Proposition 3.2. Let $f: \mathbb{P}^{1} \rightarrow \mathbb{P}^{r}$ be a general degree-d map (allowed to be degenerate if $d<r)$. Then $f^{*} T_{\mathbb{P} r}(-1)$, and consequently $f^{*} T_{\mathbb{P} r}$, satisfies interpolation.

Proof. To show $f^{*} T_{\mathbb{P} r}(-1)$ satisfies interpolation, we argue by induction on the degree $d$ of $f$; the base case $d=1$ is given by Proposition 3.1. For the inductive step $d \geq 2$, we degenerate $f: \mathbb{P}^{1} \rightarrow \mathbb{P}^{r}$ to a map $g: D \cup_{p} L \rightarrow \mathbb{P}^{r}$ from a two-component reducible rational curve; write $g_{D}=\left.g\right|_{D}$ and $g_{L}=\left.g\right|_{L}$. Assume that deg $g_{D}=d-1$ and deg $g_{L}=1$. By Lemma 2.1, it suffices to show $g^{*} T_{\mathbb{P} r}(-1)$ satisfies interpolation. Write

$$
\begin{aligned}
& \mathrm{ev}_{D}: H^{0}\left(g_{D}^{*} T_{\mathbb{P} r}(-1)\right) \rightarrow H^{0}\left(\left.T_{\mathbb{P} r}(-1)\right|_{p}\right), \\
& \mathrm{ev}_{L}: H^{0}\left(g_{L}^{*} T_{\mathbb{P} r}(-1)\right) \rightarrow H^{0}\left(\left.T_{\mathbb{P} r}(-1)\right|_{p}\right)
\end{aligned}
$$

for the natural evaluation morphisms. Pick a point $x \in L \backslash p$. Then by Lemma 2.4, it suffices to show that

$$
V=\operatorname{ev}_{D}^{-1}\left(\operatorname{ev}_{L}\left(H^{0}\left(g_{L}^{*} T_{\mathbb{P} r}(-1)(-x)\right)\right)\right) \subseteq H^{0}\left(g_{D}^{*} T_{\mathbb{P} r}(-1)\right)
$$

satisfies interpolation and has dimension

$$
\chi\left(g_{D}^{*} T_{\mathbb{P} r}(-1)\right)+\chi\left(g_{L}^{*} T_{\mathbb{P} r}(-1)(-x-p)\right)=\chi\left(g_{D}^{*} T_{\mathbb{P} r}(-1)\right)-(r-1) .
$$

Using the description of $g_{L}^{*} T_{\mathbb{P} r}$ from Proposition 3.1,

$$
V=\left\{\sigma \in H^{0}\left(g_{D}^{*} T_{\mathbb{P} r}(-1)\right):\left.\left.\sigma\right|_{p} \in T_{L}(-1)\right|_{p}\right\} .
$$

Since $\left.\left.T_{L}(-1)\right|_{p} \subseteq T_{\mathbb{p} r}(-1)\right|_{p}$ is a general subspace of codimension $r-1$, Lemma 2.5 implies $V$ satisfies interpolation and has dimension $\max \left\{0, \chi\left(g_{D}^{*} T_{\mathbb{P} r}(-1)\right)-(r-1)\right\}$. It thus suffices to note that

$$
\chi\left(g_{D}^{*} T_{\mathbb{P} r}(-1)\right)-(r-1)=d+1 \geq 0 .
$$


By inspection (or alternatively using Proposition 4.11 of [Atanasov et al. 2016]), interpolation for $f^{*} T_{\mathbb{P} r}(-1)$ implies interpolation for $f^{*} T_{\mathbb{P} r}$.

\section{Curves of higher genus}

Lemma 4.1. Let $\mathcal{E}$ be a vector bundle of rank $n$ on a reducible nodal curve $X \cup Y$, such that $X \cap Y$ is a general collection of $k$ points on $X$ (relative to $\left.\mathcal{E}\right|_{X}$ ). Suppose that $\left.\mathcal{E}\right|_{X}$ and $\left.\mathcal{E}\right|_{Y}$ satisfy interpolation. If $\chi\left(\left.\mathcal{E}\right|_{X}\right) \equiv 0 \bmod n$ and $\chi\left(\left.\mathcal{E}\right|_{X}\right) \geq n k$, then $\mathcal{E}$ satisfies interpolation.

Proof. Let $D$ be a general divisor on $X$ (in particular disjoint from $X \cap Y$ ) of degree $\left(\chi\left(\left.\mathcal{E}\right|_{X}\right)-n k\right) / n$. Write

$$
\mathrm{ev}_{X}: H^{0}\left(\left.\mathcal{E}\right|_{X}\right) \rightarrow H^{0}\left(\left.\mathcal{E}\right|_{X \cap Y}\right), \quad \mathrm{ev}_{Y}: H^{0}\left(\left.\mathcal{E}\right|_{Y}\right) \rightarrow H^{0}\left(\left.\mathcal{E}\right|_{X \cap Y}\right)
$$

for the natural evaluation morphisms, and let

$$
V=\operatorname{ev}_{Y}^{-1}\left(\operatorname{ev}_{X}\left(H^{0}\left(\left.\mathcal{E}\right|_{X}(-D)\right)\right)\right) \subseteq H^{0}\left(\left.\mathcal{E}\right|_{Y}\right)
$$

Since $\left.\mathcal{E}\right|_{X}$ satisfies interpolation and $\chi\left(\left.\mathcal{E}\right|_{X}(-D)\right)=n k$, we conclude $\mathrm{ev}_{X}$ is an isomorphism when restricted to $H^{0}\left(\left.\mathcal{E}\right|_{X}(-D)\right)$. In particular,

$$
H^{0}\left(\left.\mathcal{E}\right|_{X}(-D-X \cap Y)\right)=0,
$$

and $V=H^{0}\left(\left.\mathcal{E}\right|_{Y}\right)$ is the full space of sections. Because $\left.\mathcal{E}\right|_{Y}$ satisfies interpolation, we conclude $V$ satisfies interpolation and has dimension

$$
\chi\left(\left.\mathcal{E}\right|_{Y}\right)=\chi\left(\left.\mathcal{E}\right|_{Y}\right)+\chi\left(\left.\mathcal{E}\right|_{X}(-D-X \cap Y)\right) .
$$

This implies, via Lemma 2.4, that $\mathcal{E}$ satisfies interpolation.

Proof of Theorem 1.3. We argue by induction on $g$; the base case $g=0$ is given by Proposition 3.2. For the inductive step $g \geq 1$, we let

$$
\left(s, g^{\prime}, d^{\prime}\right)= \begin{cases}(r+2, g-r-1, d-r) & \text { if } g \geq r+1 \\ (g+1,0, d-r) & \text { otherwise }\end{cases}
$$

By construction, $g^{\prime} \geq 0$ and $1 \leq s \leq r+2$. Moreover, since $\rho(d, g, r) \geq 0$, we have either $\rho\left(d^{\prime}, g^{\prime}, r\right) \geq 0$, or $g^{\prime}=0$ and $d^{\prime} \geq 1$; and in the second case $d^{\prime} \geq s-1$.

We now degenerate $f: C \rightarrow \mathbb{P}^{r}$ to a map $g: D \cup_{\Gamma} \mathbb{P}^{1} \rightarrow \mathbb{P}^{r}$ from a two-component reducible curve; write $g_{D}=\left.g\right|_{D}$ and $g_{\mathbb{P}^{1}}=\left.g\right|_{\mathbb{P}^{1}}$. By the above, we may take $D$ to be a general curve of genus $g^{\prime}$, and $\Gamma$ a collection of $s$ points general on both $D$ and $\mathbb{P}^{1}$. We also take $g_{D}$ and $g_{\mathbb{P} 1}$ to be general maps of degrees $d^{\prime}$ and $r$ respectively 
(composing with an automorphism of $\mathbb{P}^{r}$ so that $g_{D}(\Gamma)=g_{\mathbb{P} 1}(\Gamma)$ - which exists since Aut $\mathbb{P}^{r}$ acts $(r+2)$-transitively on points in linear general position; note that $s \leq r+2$, and $g_{\mathbb{P} 1}$ is nondegenerate, while $g_{D}$ spans at least a $\left.\mathbb{P}^{\min (r, s-1)}\right)$. By Lemma 2.1, it suffices to show $g^{*} T_{\mathbb{p} r}$ satisfies interpolation.

By induction (and direct application of Proposition 3.2 in the case $g^{\prime}=0$ and $\left.d^{\prime} \geq 1\right)$, we know $g_{D}^{*} T_{\mathbb{P} r}$ and $g_{\mathbb{P} 1}^{*} T_{\mathbb{P} r}$ satisfy interpolation. Moreover, $\chi\left(g_{\mathbb{P} 1}^{*} T_{\mathbb{P} r}\right)=$ $r(r+2)$ is a multiple of $r$ which is at least $r s$. Lemma 4.1 thus yields the desired conclusion.

Proof of Theorem 1.5. We argue by induction on $g$; the base case $g=0$ is given by Proposition 3.2. For the inductive step $g \geq 1$, we write $g^{\prime}=g-1 \geq 0$ and $d^{\prime}=d-r$. Since $d-r g-1 \geq 0$, we have $d^{\prime}-r g^{\prime}-1 \geq 0$. This in turn implies either $\rho\left(d^{\prime}, g^{\prime}, r\right) \geq 0$, or $g^{\prime}=0$ and $d^{\prime} \geq 1$.

We now degenerate $f: C \rightarrow \mathbb{P}^{r}$ to a map $g: D \cup_{\Gamma} \mathbb{P}^{1} \rightarrow \mathbb{P}^{r}$ from a two-component reducible curve; write $g_{D}=\left.g\right|_{D}$ and $g_{\mathbb{P} 1}=\left.g\right|_{\mathbb{P 1}}$. By the above, we may take $D$ to be a general curve of genus $g^{\prime}$, and $\Gamma$ a collection of 2 points general on both $D$ and $\mathbb{P}^{1}$. We also take $g_{D}$ and $g_{\mathbb{P} 1}$ to be general maps of degrees $d^{\prime}$ and $r$ respectively (composing with an automorphism of $\mathbb{P}^{r}$ so that $g_{D}(\Gamma)=g_{\mathbb{P}^{1}}(\Gamma)$ - which exists since Aut $\mathbb{P}^{r}$ acts 2-transitively). By Lemma 2.1, it suffices to show $g^{*} T_{\mathbb{P} r}(-1)$ satisfies interpolation.

By induction (and direct application of Proposition 3.2 in the case $g^{\prime}=0$ and $\left.d^{\prime} \geq 1\right)$, we know $g_{D}^{*} T_{\mathbb{P} r}(-1)$ and $g_{\mathbb{P} 1}^{*} T_{\mathbb{P} r}(-1)$ satisfy interpolation. Moreover, $\chi\left(g_{\mathbb{P} 1}^{*} T_{\mathbb{P} r}(-1)\right)=2 r$. Lemma 4.1 thus yields the desired conclusion.

\section{Acknowledgements}

The author would like to thank Joe Harris for his guidance throughout this research, as well as Atanas Atanasov and David Yang for helpful conversations. The author would also like to acknowledge the generous support both of the Fannie and John Hertz Foundation, and of the Department of Defense (NDSEG fellowship).

\section{References}

[Atanasov 2014] A. Atanasov, "Interpolation and vector bundles on curves", preprint, 2014. arXiv [Atanasov et al. 2016] A. Atanasov, E. Larson, and D. Yang, "Interpolation for normal bundles of general curves", preprint, 2016. arXiv

[Griffiths and Harris 1980] P. Griffiths and J. Harris, "On the variety of special linear systems on a general algebraic curve”, Duke Math. J. 47:1 (1980), 233-272. MR Zbl

Communicated by Ravi Vakil

Received 2015-11-30

Accepted 2016-05-16

elarson3@gmail.com

Massachusetts Institute of Technology, 77 Massachusetts Avenue, Cambridge, MA 02139-4307, United States 


\section{Algebra \& Number Theory}

msp.org/ant

\section{EDITORS}

MANAGING EDITOR

Bjorn Poonen

Massachusetts Institute of Technology

Cambridge, USA

\author{
EDITORIAL BOARD CHAIR \\ David Eisenbud \\ University of California \\ Berkeley, USA
}

BOARD OF EDITORS

Georgia Benkart

Dave Benson

Richard E. Borcherds

John H. Coates

J-L. Colliot-Thélène

Brian D. Conrad

Hélène Esnault

Hubert Flenner

Sergey Fomin

Edward Frenkel

Andrew Granville

Joseph Gubeladze

Roger Heath-Brown

Craig Huneke

Kiran S. Kedlaya

János Kollár

Yuri Manin

Philippe Michel
University of Wisconsin, Madison, USA

University of Aberdeen, Scotland

University of California, Berkeley, USA

University of Cambridge, UK

CNRS, Université Paris-Sud, France

Stanford University, USA

Freie Universität Berlin, Germany

Ruhr-Universität, Germany

University of Michigan, USA

University of California, Berkeley, USA

Université de Montréal, Canada

San Francisco State University, USA

Oxford University, UK

University of Virginia, USA

Univ. of California, San Diego, USA

Princeton University, USA

Northwestern University, USA

École Polytechnique Fédérale de Lausanne
Susan Montgomery

Shigefumi Mori

Raman Parimala

Jonathan Pila

Anand Pillay

Victor Reiner

Peter Sarnak

Joseph H. Silverman

Michael Singer

Vasudevan Srinivas

J. Toby Stafford

Ravi Vakil

Michel van den Bergh

Marie-France Vignéras

Kei-Ichi Watanabe

Efim Zelmanov

Shou-Wu Zhang
University of Southern California, USA

RIMS, Kyoto University, Japan

Emory University, USA

University of Oxford, UK

University of Notre Dame, USA

University of Minnesota, USA

Princeton University, USA

Brown University, USA

North Carolina State University, USA

Tata Inst. of Fund. Research, India

University of Michigan, USA

Stanford University, USA

Hasselt University, Belgium

Université Paris VII, France

Nihon University, Japan

University of California, San Diego, USA

Princeton University, USA

\section{PRODUCTION}

production@msp.org

Silvio Levy, Scientific Editor

See inside back cover or msp.org/ant for submission instructions.

The subscription price for 2016 is US $\$ 290 /$ year for the electronic version, and $\$ 485 /$ year ( $+\$ 55$, if shipping outside the US) for print and electronic. Subscriptions, requests for back issues and changes of subscribers address should be sent to MSP.

Algebra \& Number Theory (ISSN 1944-7833 electronic, 1937-0652 printed) at Mathematical Sciences Publishers, 798 Evans Hall \#3840, c/o University of California, Berkeley, CA 94720-3840 is published continuously online. Periodical rate postage paid at Berkeley, CA 94704, and additional mailing offices.

ANT peer review and production are managed by EditFLOW ${ }^{\circledR}$ from MSP.

\section{PUBLISHED BY}

- mathematical sciences publishers

nonprofit scientific publishing

http://msp.org/

(C) 2016 Mathematical Sciences Publishers 


\section{Algebra \& Number Theory}

Volume $10 \quad$ No. $4 \quad 2016$

Moduli of morphisms of logarithmic schemes

JONATHAN WISE

Residual intersections and the annihilator of Koszul homologies

SEYED HAMID HaSSANZADEH and Jose NAÉLITON

The Prym map of degree-7 cyclic coverings

HERBERT LANGE and ANGELA ORTEGA

SIMON MARSHALL

Hasse principle for Kummer varieties

Yonatan HaRPaZ and AlEXEI N. SkoRobogatov

Analytic continuation on Shimura varieties with $\mu$-ordinary locus

STÉPHANE BIJAKOWSKI

A note on secondary $K$-theory

GONÇALO TABUADA

Nef cones of Hilbert schemes of points on surfaces

Barbara Bolognese, Jack Huizenga, Yinbang Lin, Eric Riedl,

BENJAMIN SCHMidT, MATTHEW WoOlF and XiaOlei ZHAO

Interpolation for restricted tangent bundles of general curves 